\title{
Multi-objective optimization of a type of ellipse-parabola shaped superelastic flexure hinge
}

\author{
Zhijiang Du, Miao Yang, and Wei Dong \\ State Key Laboratory of Robotics and System, Harbin Institute of Technology, 2 Yikuang Street, Harbin, \\ 150080, China \\ Correspondence to: Wei Dong (dongwei@hit.edu.cn)
}

Received: 12 January 2016 - Revised: 21 March 2016 - Accepted: 20 April 2016 - Published: 12 May 2016

\begin{abstract}
Flexure hinges made of superelastic materials is a promising candidate to enhance the movability of compliant mechanisms. In this paper, we focus on the multi-objective optimization of a type of ellipse-parabola shaped superelastic flexure hinge. The objective is to determine a set of optimal geometric parameters that maximizes the motion range and the relative compliance of the flexure hinge and minimizes the relative rotation error during the deformation as well. Firstly, the paper presents a new type of ellipse-parabola shaped flexure hinge which is constructed by an ellipse arc and a parabola curve. Then, the static responses of superelastic flexure hinges are solved via non-prismatic beam elements derived by the co-rotational approach. Finite element analysis (FEA) and experiment tests are performed to verify the modeling method. Finally, a multi-objective optimization is performed and the Pareto frontier is found via the NSGA-II algorithm.
\end{abstract}

\section{Introduction}

Flexure hinges is a substitution of conventional rotational joint in precision engineering. The monolithic structure brings flexure hinge various superior properties, e.g. high resolution, fine precision, compact structure, and the elimination of friction and lubrication (Paros and Weisbord, 1965). Therefore, flexure hinges are widely employed in micro positioning stages, precision alignment devices, micro manipulators, scanning electron microscopy and antennas where high precision and high resolution are required (Pham and Chen, 2005; Yong et al., 2008; Du et al., 2014). However, despite all the advantages aforementioned, flexure hinges also have some inherent shortcomings, for example the limited motion range restricted by the allowable stress and strain of the material. In addition, the rotational center shift also reduces the absolute accuracy of flexure hinge based compliance mechanisms.

The movability of a flexure hinge comes from the deformation of its structure. The 3-D sketch of a flexure hinge is shown in Fig. 1, it can be seen that the deformation almost exclusively takes place in the weakest region. In order to avoid plastic deformation the movability of the flexure hinges are severely restricted (Friedrich et al., 2014). A promising ap- proach to enhance their motion capacity is to use superelastic materials, e.g. shape memory alloy (SMA), to fabricate flexure hinges since they can provide much larger allowable strains. The maximum recoverable strain of SMA is about $8 \%$, while for the most frequently used materials to fabricate flexure hinges, e.g. steel or aluminum, the maximum recoverable strain is $0.2-0.4 \%$ (Desroches and Delemont, 2002). As it is mentioned in Hesselbach and Raatz (2000), the motion range of a flexure hinge made of SMA can reach as much as $30^{\circ}$, which can satisfy most of the application requirements for revolution joints in parallel mechanisms.

However, formulating a static deformation model for the superelastic flexure hinge is a challenging work. Although the flexure hinges can be modeled as Euler beams since the minimum thickness of a planar flexure hinge is much less than the hinge length (Chen et al., 2011), nonlinearities introduced by large deformation and the constitutive relationship of superelastic material made it extremely difficult to obtain analytic solutions to describe the static response of the flexure hinges. Previous modeling methods, like the integration of beam theory (Wu and Zhou, 2002), Castigliano's second theorem (Lobontiu et al., 2002a), and pseudo rigid body method (Kim et al., 2013) are also infeasible to solve 


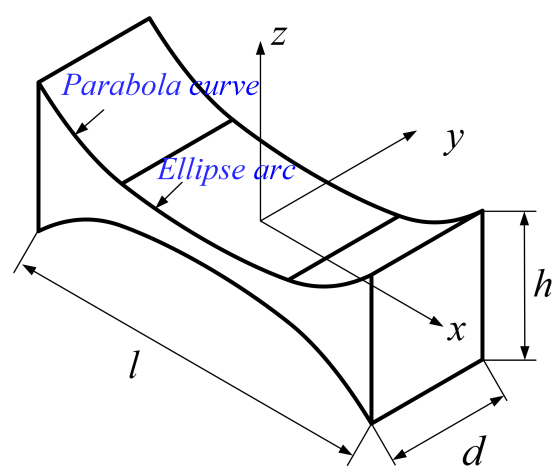

Figure 1. The 3-D model of the proposed ellipse-parabola shaped flexure hinge.

this problem. In this paper, we will use nonlinear finite element method (FEM) to formulate the relationship between the deformation and loads of superelastic flexure hinges.

The performance of a flexure hinge is completely determined by its notch shape, once the material of the flexure hinge is chosen. Various types of flexure hinges with different shapes have been investigated in the past years, e.g. V-shaped flexure hinges (Tian et al., 2010), parabolic and hyperbolic flexure hinges (Lobontiu et al., 2002b; Chen et al., 2009), elliptical-arc-fillet flexure hinges (Chen et al., 2008), flexure hinges that considered the machining errors (Shi et al., 2013), and even the exponent-sine-shaped flexure hinges (Wang et al., 2013). But these flexure hinges defined by only few parameters that restrict the degree of freedom to choose optimal shapes of flexure hinges. De Bona and Gh Munteanu (2005) illustrated a flexure hinge constructed by a group of cubic-spline curves which maximized the probability to find out the optimal flexure hinges, however it needs 9 parameters to formulate the shape, which will increase the computational complexity of the optimization dramatically, especially for flexure hinges made of superelastic materials.

To obtain a balance between the diversity of the optimization and the computational complexity, this paper proposed a new type of ellipse-parabola shaped flexure hinge which is constructed by an ellipse arc and a parabola curve. The parameters that defining the profile of the ellipse-parabola shaped flexure hinge are the notch length, the minimum thickness, the ellipticity of the ellipse arc and the terminated eccentric angle. It can describe a large range of flexure hinges by change the values of the parameters and all the parameters have certain meanings in geometry.

The multi-objective optimization is performed for the ellipse-parabola shaped superelastic flexure hinge to obtain excellent comprehensive performances. The remainder of this paper is organized as follows. Section 2 gives a description of the proposed ellipse-parabola shaped flexure hinge. Section 3 establishes the static deformation model of the flexure hinge by using nonlinear beam elements derived by the

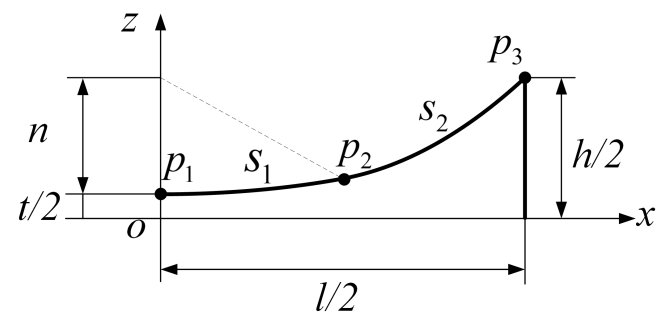

Figure 2. The 2-D schematic of the ellipse-parabola shaped flexure hinge.

co-rotational approach. The deformation model has been verified by FEA and experiment test in Sect. 4. In Sect. 5, the multi-objective optimization of the ellipse-parabola shaped superelastic flexure hinge is formulated and the Pareto frontier is found via the NSGA-II algorithm. Finally, the conclusions of this paper is presented in Sect. 6 .

\section{Structure of the ellipse-parabola shaped flexure hinge}

The elliptic flexure hinge has relative larger motion range than the parabolic flexure hinge and the hyperbolic flexure hinge, and the parabolic flexure hinge has a higher transmission accuracy than the elliptic flexure hinge (Lobontiu et al., 2002a). Better comprehensive performances of a flexure hinge can be obtained by combining the two profiles together. The proposed ellipse-parabola shaped flexure hinge is shown in Fig. 1.

It can be seen that the ellipse-parabola shaped flexure hinge is fully symmetric and $o-x y z$ is the corresponding Cartesian coordinate, the origin is located at the center of the structure, $x$ axis follows with the longitudinal axis of symmetry and $z$ axis coincides with the transverse axis. A 2-D sketch of the flexure hinge is illustrated in Fig. 2. The profile consists of two parts, i.e. an ellipse arc and a parabola curve, the two curves are smoothly connected at the intersection $p_{2}$. Moreover, $l$ denotes the notch length of the flexure hinge, $t$ denotes the minimum thickness, and $h$ and $d$ are the height and width of the flexure hinge respectively.

Curve $s_{1}$ is an ellipse arc with its center is located at $\left(0, \frac{h}{2}\right)$, and it can be represented in a parametric form as below,

$\left\{\begin{array}{l}x(\varphi)=n e \cos \varphi \\ z(\varphi)=n \sin \varphi+\frac{h}{2}\end{array} \varphi_{\mathrm{t}} \leq \varphi \leq 0\right.$ and $-\frac{\pi}{2} \leq \varphi_{\mathrm{t}} \leq 0$,

where $n=\frac{h-t_{\mathrm{S}}}{2}$ is the vertical semi-axe, $e$ is the ellipticity of the ellipse arc which is the quotient of the horizontal semiaxe and the vertical semi-axe, the parameter $\varphi$ is the eccentric angle, and $\varphi_{\mathrm{t}}$ is the terminated eccentric angle at $p_{2}$.

The parabola curve $s_{2}$ passes through $p_{2}=$ $\left(n e \cos \varphi_{\mathrm{t}}, n \sin \varphi_{\mathrm{t}}+\frac{h}{2}\right)$ and $p_{3}=\left(\frac{l}{2}, \frac{h}{2}\right)$, and have the same slope with $s_{1}$ at point $p_{2}$. Then, the thickness of the flexure hinge in $s_{2}$ can be formulated by applying the 


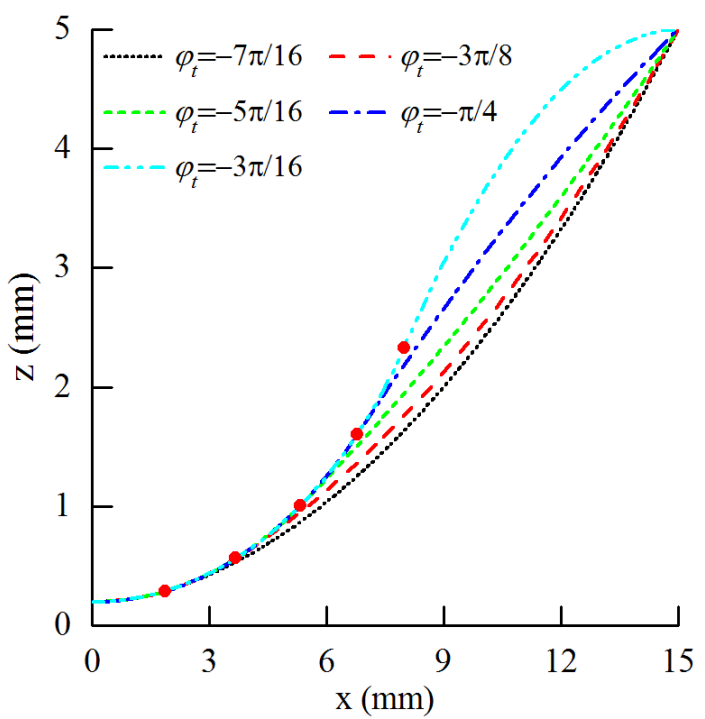

Figure 3. Notch shapes with different $\varphi_{\mathrm{t}}\left(e=2\right.$ and $\varphi_{\mathrm{t}}$ ranges from $-\frac{7 \pi}{16}$ to $\left.-\frac{3 \pi}{16}\right)$.

Hermite interpolation.

$z(x)=a\left(x-n e \cos \varphi_{\mathrm{t}}\right)^{2}+b\left(x-n e \cos \varphi_{\mathrm{t}}\right)+c$.

The coefficients in Eq. (2) are given by

$a=\frac{2 l \cos \varphi_{\mathrm{t}}-4 n e}{e \sin \varphi_{\mathrm{t}}\left(l-2 n e \cos \varphi_{\mathrm{t}}\right)^{2}}, \quad b=-\frac{1}{e \tan \varphi}$, and $c=\left(\frac{h}{2}+n \sin \varphi_{\mathrm{t}}\right)$.

To further illustrate the shape of the proposed ellipseparabola shaped flexure hinge, suppose that $l=30 \mathrm{~mm}$, $t_{\mathrm{s}}=0.4 \mathrm{~mm}$, and $h=10 \mathrm{~mm}$, then the profile is fully determined by $e$ and $\varphi_{\mathrm{t}}$. Figure 3 shows the profiles of the flexure hinges with different $\varphi_{\mathrm{t}}$, where the ellipticity $e=2, \varphi_{\mathrm{t}}$ ranges from $-\frac{7 \pi}{16}$ to $-\frac{3 \pi}{16}$, and the red dots in the figure are the intersections, i.e. $p_{2}$. It can be seen that the percentage of $s_{1}$ in the whole profile increases with the increase of $\varphi_{\mathrm{t}}$. On the other hand, for a given $\varphi_{\mathrm{t}}=-\frac{\pi}{3}$, and $e$ ranges from 1 to 3 , the profiles are shown in Fig. 4, it can be seen that the flexure hinges become more flat with the increase of $e$, thus the effective deform length increases.

\section{Modeling of the superelastic flexure hinges}

The ellipse-parabola shaped superelastic flexure hinge can be considered as a cantilever beam with variable cross section, geometric and material nonlinearities. In this paper, we use the nonlinear FEM to model deformation of the flexure hinge. The co-rotational approach provides an effective way to derive beam elements with geometric nonlinearity. By introducing a local coordinate system (LCS) attached on the

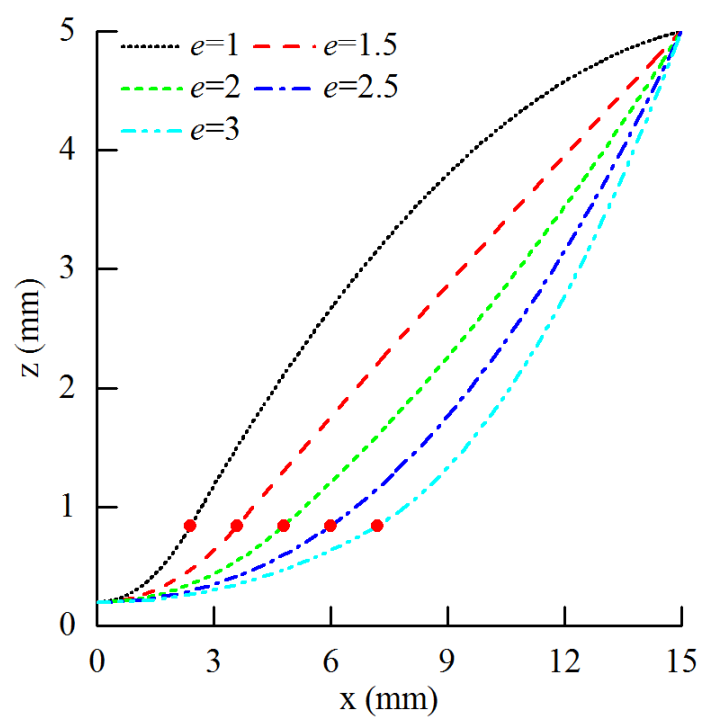

Figure 4. Notch shapes with different $e\left(\varphi_{\mathrm{t}}=-\frac{\pi}{3}\right.$ and $e$ ranges from 12 to 20 ).

element, the displacement and the deformation of the element is separated, and then a simple form of the local tangent stiffness matrix and internal force vector of the element are obtained. The co-rotational method used here is similar to those in Pacoste and Eriksson (1997) and Criesfield (1991), and briefly described in the following discussion.

Figure 5 shows a two-node beam element in the initial and current configurations. The coordinate of the two nodes in the global coordinate system (GCS) $x o z$ are $\left(x_{1}, y_{1}\right)$ and $\left(x_{2}\right.$, $\left.y_{2}\right)$ initially, and the global nodal displacement vector of the element is given by

$\boldsymbol{q}_{\mathrm{g}}=\left\{u_{1}, w_{1}, \theta_{1}, u_{2}, w_{2}, \theta_{2}\right\}^{T}$,

where $u$ and $w$ are nodal displacements in the $x$ and $z$ direction respectively; $\theta$ is the nodal rotation angle, and the subscripts 1 and 2 refer to the nodes 1 and 2 .

The LCS $x_{l} o_{l} z_{l}$ is assigned as that its origin is located at node 1 and $x$ axis directs to node 2 . Thus, the local displacement vector of the element contains only 3 components shown as below

$\boldsymbol{q}_{1}=\left\{\bar{u}, \bar{\theta}_{1}, \bar{\theta}_{2}\right\}^{T}$.

Based on the kinematics depicted in Fig. 5, the axis displacement and rotation angles of the two nodes in LCS can be obtained

$\bar{u}=L-L_{0}$
$\bar{\theta}_{1}=\theta_{1}-\alpha$
$\bar{\theta}_{2}=\theta_{2}-\alpha$

where $L_{0}$ and $L$ are the initial and current length of the element respectively, and $\alpha$ is the rigid rotational displacement 


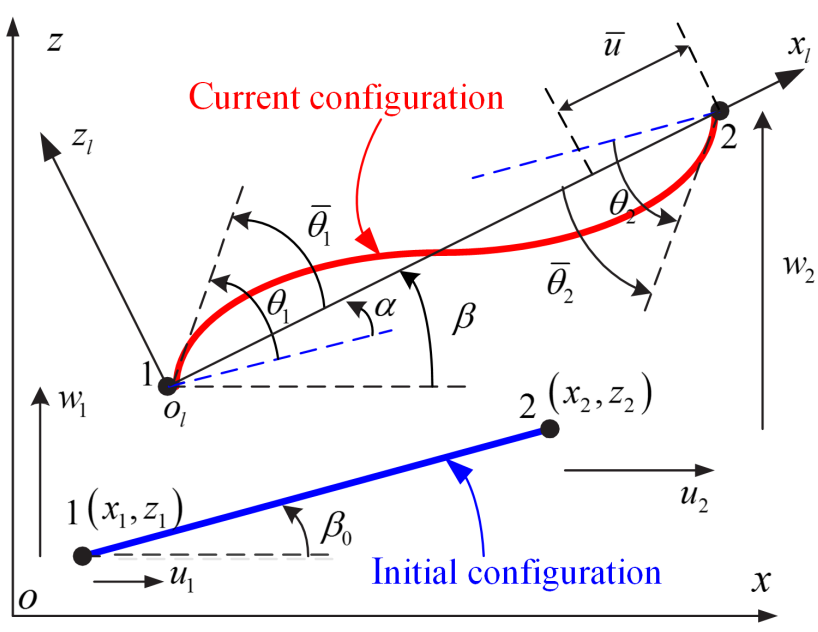

Figure 5. Beam kinematics.

of the element and formulated as below,

$$
\begin{aligned}
& L_{0}=\sqrt{\left(x_{2}-x_{1}\right)^{2}+\left(z_{2}-z_{1}\right)^{2}} \\
& L=\sqrt{\left(x_{2}+u_{2}-x_{1}-u_{1}\right)^{2}+\left(z_{2}+w_{2}-z_{1}-w_{1}\right)^{2}} \\
& \alpha=\beta-\beta_{0}=\arctan \left(\frac{z_{2}+w_{2}-z_{1}-w_{1}}{x_{2}+u_{2}-x_{1}-u_{1}}\right)-\arctan \left(\frac{z_{2}-z_{1}}{x_{2}-x_{1}}\right)
\end{aligned}
$$

Taking a differentiation of the Eq. (5) on both side, the relationship between the local virtual displacement $\delta \boldsymbol{q}_{1}$ and the global virtual displacement $\delta \boldsymbol{q}_{\mathrm{g}}$ can be expressed as

$\delta \boldsymbol{q}_{1}=\mathbf{B} \delta \boldsymbol{q}_{\mathrm{g}}$,

where $\mathbf{B}$ is the transformation matrix.

Since the virtual work of the element is equivalent in both LCS and GCS, the global internal nodal force vector $\boldsymbol{f}_{\mathrm{g}}$ can be formulated as

$\boldsymbol{f}_{\mathrm{g}}=\mathbf{B}^{T} \boldsymbol{f}_{1}$,

where $f_{1}=\left\{\bar{N}, \bar{M}_{1}, \bar{M}_{2}\right\}$ is the local internal nodal force vector.

By taking the differentiation of Eq. (8) with respect to the global nodal displacement, the global tangent stiffness matrix is obtained

$\mathbf{K}_{\mathrm{g}}=\frac{\partial \boldsymbol{f}_{\mathrm{g}}}{\partial \boldsymbol{q}_{\mathrm{g}}}=\mathbf{B}^{T} \mathbf{K}_{\mathrm{l}} \mathbf{B}+\frac{\bar{N}}{L} \mathbf{z z}^{T}+\frac{\bar{M}_{1}+\bar{M}_{2}}{L^{2}}\left(\mathbf{z r}^{T}+\mathbf{r z}^{T}\right)$,

where $\mathbf{K}_{1}$ is the local stiffness matrix and defined by $\delta \boldsymbol{f}_{1}=$ $\mathbf{K}_{1} \delta \boldsymbol{q}_{1}, \mathbf{r}$ and $\mathbf{z}$ are transformation matrices $\mathbf{r}=\mathbf{b}_{1}^{T}, \mathbf{z}=\frac{\partial \mathbf{r}}{\partial \beta}$, and $\mathbf{b}_{1}$ is the first row of matrix $\mathbf{B}$.

Based on the Euler-Bernoulli beam theory, the axial strain in a beam element for large deformation is given by

$\varepsilon(\bar{x}, \bar{z})=\frac{\partial u_{0}(\bar{x})}{\partial \bar{x}}+\frac{1}{2}\left(\frac{\partial w_{0}(\bar{x})}{\partial \bar{x}}\right)^{2}-\bar{z} \frac{\partial^{2} w_{0}(\bar{x})}{\partial \bar{x}^{2}}$, where $u_{0}(\bar{x})$ and $w_{0}(\bar{x})$ are the axial and transverse displacement on the beam mid-plane respectively. Applying the classical linear and cubic interpolation for the axial displacement $u_{0}(\bar{x})$ and transverse displacement $w_{0}(\bar{x})$ respectively, the axial displacement $u_{0}(\bar{x})$ can be then rewritten as

$u_{0}=\frac{\bar{x}}{L} \bar{u}$

and the transverse displacement $w_{0}(\bar{x})$ is given as

$w_{0}=\left(\bar{x}-2 \frac{\bar{x}^{2}}{L}+\frac{\bar{x}^{3}}{L^{2}}\right) \bar{\theta}_{1}+\left(-\frac{\bar{x}^{2}}{L}+\frac{\bar{x}^{3}}{L^{2}}\right) \bar{\theta}_{2}$.

Considering the membrane locking involved in this problem, the first two items in Eq. (10) are replaced by the average axial strain which is defined as following

$\varepsilon_{\mathrm{ma}}=\frac{1}{L} \int_{0}^{L}\left[\frac{\partial u_{0}}{\partial \bar{x}}+\frac{1}{2}\left(\frac{\partial w_{0}}{\partial \bar{x}}\right)^{2}\right] \mathrm{d} \bar{x}$.

Computing the integration in Eq. (13) and combine with Eq. (10), the axial strain $\varepsilon$ can be rewritten as

$$
\begin{aligned}
\varepsilon= & \frac{\bar{u}}{L}+z\left(\left(\frac{4}{L}-\frac{6 x}{L^{2}}\right) \bar{\theta}_{1}+\left(\frac{2}{L}-\frac{6 x}{L^{2}}\right) \bar{\theta}_{2}\right)+\frac{1}{15} \bar{\theta}_{1}^{2} \\
& +\frac{1}{30} \bar{\theta}_{1} \bar{\theta}_{2}+\frac{1}{15} \bar{\theta}_{2}^{2}
\end{aligned}
$$

The local internal forces are then calculated by applying principle of virtual work.

$V=\int_{\mathrm{v}} \sigma \delta \varepsilon \mathrm{d} v=N \delta \bar{u}+M_{1} \delta \bar{\theta}_{1}+M_{2} \delta \bar{\theta}_{2}$.

Superelasticity of SMA refers to that the material can undergo very large non-elastic strains and get fully recovered by unloading because of the phase transportation of the material's microstructure (Liew et al., 2004). The uniaxial isothermal stress-strain curve of Nitinol (the most frequently used SMA) measured by experiment is presented in Fig. 6. To describe the nonlinear relationship between the stress and strain of the material, a bilinear constitutive model is adopted in this paper.

$\sigma= \begin{cases}E \varepsilon & \left(\varepsilon \leq \varepsilon_{\mathrm{s}}\right) \\ \sigma_{\mathrm{s}}+E_{1}\left(\varepsilon-\varepsilon_{\mathrm{s}}\right) & \left(\varepsilon>\varepsilon_{\mathrm{s}}\right)\end{cases}$

where $E$ is the Young's modulus of the material in the elastic stage, $E_{1}$ is the modulus in phase transformation, $\varepsilon_{\mathrm{s}}$ and $\sigma_{\mathrm{s}}$ are the strain and stress at the start point of transformation respectively, $\varepsilon_{\mathrm{f}}$ and $\sigma_{\mathrm{f}}$ are the strain and stress at the finial point of transformation.

Substituting Eqs. (14) and (16) into Eq. (15) and separating variables yield the local internal forces of the element. 


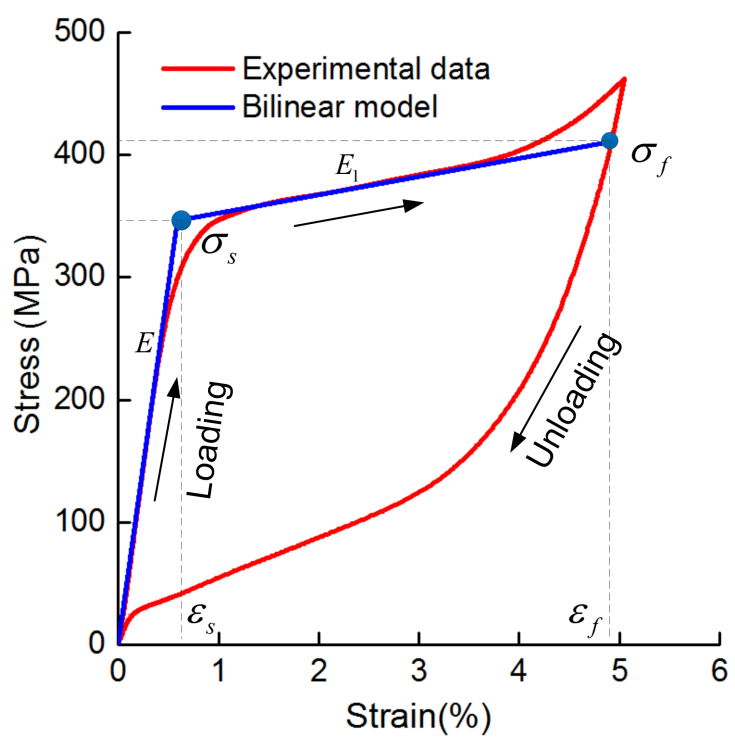

Figure 6. Experimental data of Nitinol uniaxial tensile behavior and the bilinear constitutive model.

And the local tangent stiffness matrix is than obtained by taking differential of the local internal force with respect to the components of $\boldsymbol{q}_{1}$ respectively

$\mathbf{K}_{1_{i, j}}=\frac{\partial \boldsymbol{f}_{1 i}}{\partial \boldsymbol{q}_{1 j}}(i=1 \ldots 3, j=1 \ldots 3)$.

Combining Eqs. (15)-(17) with Eq. (9), the global tangent stiffness matrix of the element $\mathbf{K}_{\mathrm{g}}$ and the global internal force vector $\boldsymbol{F}_{\mathrm{g}}$ of the beam element can be obtained.

The derived global force vector $\boldsymbol{F}_{\mathrm{g}}$ and global tangent stiffness matrix $\mathbf{K}_{\mathrm{g}}$ of the element are then assembled to construct equilibrium equations of the structure. The system of nonlinear equations is solved by a load control algorithm. And the Newton-Raphson method is used to obtain the nodal displacements that minimize the residual stress during every incremental load step. A program implementing this algorithm has been written in Matlab. The end displacements and the stress and strain on the structure can be easily acquired by the program.

\section{Verification}

To validate the proposed co-rotational beam element based model (CRM), both finite element analysis by ANSYS (AFE) and experimental tests (EXP) are conducted. The height and the width of the flexure hinge samples are designed as $h=10 \mathrm{~mm}$ and $d=5 \mathrm{~mm}$. The corresponding parameters of the material obtained from experimental measurement are as follow: $E=58.51 \mathrm{GPa}, E_{1}=1.5 \mathrm{GPa}$, $\sigma_{\mathrm{s}}=346 \mathrm{MPa}, \sigma_{\mathrm{f}}=410 \mathrm{MPa}$.

The AFE model of the ellipse-parabola shaped superelastic flexure hinge is established via the APDL language and

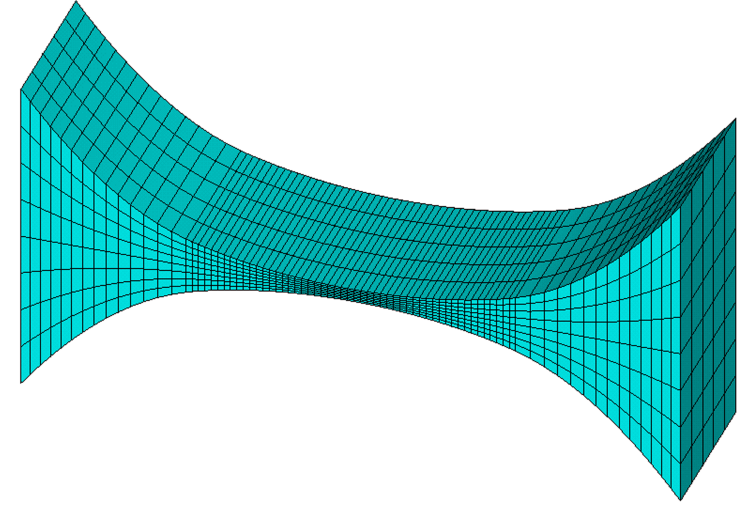

Figure 7. The finite element model in ANSYS.

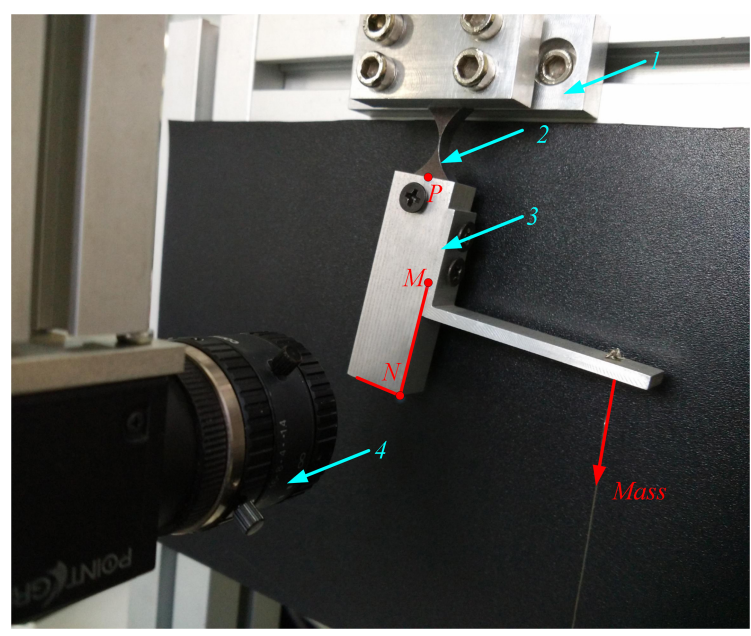

Figure 8. The experiment setup. (1: the base; 2 : the flexure hinge sample; 3: the loading device; 4: the CCD camera)

discretized by 20 -node solid 186 elements in a mapped meshing way as shown in Fig. 7. The bilinear constitutive model is introduced into the model by the TB command. And the flexure hinge is fixed on one end, and loaded with a moment $M_{y}$ on the other end.

The photograph of the experiment setup is illustrated in Fig. 8. The deformation of the flexure hinge samples are obtained by a computer vision measurement system. The CCD camera (PointGrey BFLY-PGE-50H5M-C) with $2448 \times 2048$ pixels is used and the measurement area is about $20 \mathrm{~mm} \times$ $16 \mathrm{~mm}$. Thus the resolution of the vision measurement is better than $8 \mu \mathrm{m}$ and $1 \mathrm{mrad}$ for transverse displacement and rotational angle respectively.

Three sample flexure hinges are manufactured by WEDM and deformed by standard weights via the loading device. The moment introduced by the loading device is also taken into consideration to calculate the deformation of the flexure hinge. Transverse displacement of point $N$ and slope of line $\mathrm{MN}$ are obtained by the computer vision measurement system. For easy to comparison, the transverse and ro- 
tational displacement of point $P$ obtained by CRM and AFE are transformed into the deformation information of point $N$ through a geometric transformation.

The result obtained by different methods are listed in Table 1 , where $w$ is the transverse displacements at point $N$, and $\theta$ is the rotational angle. It can be seen that the results calculated by CRM agree well with those by AFE and EXP, and the maximum relative errors are within 1.5 and $7 \%$ respectively. The deviation between the CRM and the EXP may be caused by the approximation of the constitutive model and the manufacturing error of the flexure hinge.

It should be pointed out that the AFE model consists of approximately 3200 elements, and consumes more than $350 \mathrm{~s}$ to calculate the results, while the CRM contains only 20 elements and complete the solution within $5 \mathrm{~s}$ which is almost 70 times faster than AFE. It will be really helpful for the optimization of superelastic flexure hinges which will be discussed in the following section.

\section{Multi-objective optimization of the superelastic flexure hinge}

\subsection{Optimization objectives}

The flexure hinge is designed to serve as a rotational joint with its rotation center is located at the geometric center of the thinnest region. But it does not behave exactly as the ideal rotational joint. Considering the characteristics of the ellipse-parabola shaped superelastic flexure hinge, three performance indexes have been defined.

Motion range $\boldsymbol{\theta}_{\max }$ The motion range $\theta_{\max }$ of an ellipseparabola shaped superelastic flexure hinge is defined as the rotational angle between its two end sections, when the maximum strain on the structure up to the admissible strain. Though the maximum recoverable strain of the Nitinol used in this paper is about $4.9 \%$ as shown in Fig. 6, we set the allowable strain as $3 \%$ in this paper to obtain a long life cycle.

Relative rotational error $\gamma$ The trace at the free end of the ellipse-parabola shaped superelastic flexure hinge is not a perfectly circular arc, because the rotation center of the flexure hinge significantly shifts during the deformation. This may introduce undesirable errors for transmission. The relative rotational error is defined as,

$\gamma=\frac{\Delta r}{\theta_{\max }}$,

where $\Delta r$ is the deviation between the flexure hinge and an ideal rotational joint at the maximum rotation angle $\theta_{\max }$, and it is given by

$$
\Delta r=\sqrt{\left[u+\frac{l}{2}\left(1-\cos \theta_{\max }\right)\right]^{2}+\left(w-\frac{l}{2} \sin \theta_{\max }\right)^{2}} .
$$

Relative compliance $\rho$ The flexure hinges should be very compliant in the motion axis and relative stiff in other directions, therefore the relative compliance is defined as:

$\rho=\frac{C_{\mathrm{m}}}{C_{\mathrm{f}}}$,

where $C_{\mathrm{m}}$ and $C_{\mathrm{f}}$ are the angular compliance about $y$ axis and the linear compliance along $x$ axis respectively, and they can be defined as

$$
C_{\mathrm{m}}=\frac{\theta_{\max }}{M_{\mathrm{ymax}}}, C_{\mathrm{f}}=\frac{1}{\mathrm{~d} E} \int_{0}^{l} \frac{1}{2 z(x)} \mathrm{d} x,
$$

in which $M_{\text {ymax }}$ is the moment loaded at the free end of the superelastic flexure hinge when $\theta_{\max }$ is reached.

The optimization problem aims to determine a set of optimal geometric parameters of the superelastic flexure hinge that minimize the difference between a superelastic flexure hinge and an ideal rotational joint, i.e. maximize the motion range and the relative compliance and minimize the relative rotation error during the deformation as well. Thus, the optimization objectives can be described as:

$f(\boldsymbol{x})=\left[-\theta_{\max }, \gamma,-\rho\right] \rightarrow \min$.

\subsection{Design variables and optimization constraints}

The ellipse-parabola shaped superelastic flexure hinge investigated in this paper is made of a rectangular Nitinol strip by removing two symmetric cutouts. Among the parameters that define the geometry of the flexure hinge, the height $h$ and width $d$ are determined by the Nitinol strip and the minimum thickness of the hinge $t$ is determined by the processing method. In order to optimize the ellipse-parabola shaped superelastic flexure hinge, three design variables are introduced: the length of flexure hinge $l$, the ellipticity of the ellipse arc $e$ and the eccentric angle $\varphi_{\mathrm{t}}$ at $p_{2}$. Hence, the design parameters vector $\boldsymbol{x}$ is given by:

$\boldsymbol{x}=\left[l, e, \varphi_{\mathrm{t}}\right]$.

According the definition of the flexure hinge profile two geometric constraints have to be added. The first one is related to the $x$ coordinate of point $p_{2}$,

$g_{1}(\boldsymbol{x})=n e \cos \varphi_{\mathrm{t}}-\frac{l}{2} \leq 0$.

And the second constraint is to make sure that the curve is monotonous, thus the slop of the profile at $p_{3}$ must be positive

$g_{2}(\boldsymbol{x})=-2 a\left(\frac{l}{2}-n e \cos \varphi_{\mathrm{t}}\right)-b \leq 0$.

The boundary constraints in this optimization problem are defined as below

$5 \leq l \leq 40 ; 1 \leq e \leq 5 ;-\frac{\pi}{2} \leq \varphi_{\mathrm{t}} \leq 0$. 
Table 1. Displacements calculated by CRM, AFE and EXP.

\begin{tabular}{ccccccccc}
\hline $\begin{array}{c}l \\
(\mathrm{~mm})\end{array}$ & $e$ & $\begin{array}{c}\varphi_{\mathrm{t}} \\
(\mathrm{rad})\end{array}$ & $\begin{array}{c}M_{y} \\
(\mathrm{Nmm})\end{array}$ & Method & $\begin{array}{c}w \\
(\mathrm{~mm})\end{array}$ & $\begin{array}{c}\text { Error } \\
(\%)\end{array}$ & $\begin{array}{c}\theta \\
\left({ }^{\circ}\right)\end{array}$ & $\begin{array}{c}\text { Error } \\
(\%)\end{array}$ \\
\hline \multirow{2}{*}{15} & 3 & $-\frac{7}{16} \pi$ & \multirow{2}{*}{71.024} & FEM & 17.8665 & 1.1695 & 18.5121 & 1.1440 \\
& & & EXP & 18.8895 & 6.5217 & 19.6255 & 6.7528 \\
\hline \multirow{2}{*}{20} & \multirow{2}{*}{2} & $-\frac{1}{3} \pi$ & \multirow{2}{*}{71.488} & FEM & 13.2491 & 0.5550 & 12.9546 & 0.5750 \\
& & & & EXP & 13.7327 & 4.0567 & 13.4359 & 4.1365 \\
\hline \multirow{2}{*}{25} & \multirow{2}{*}{1.5} & $-\frac{1}{4} \pi$ & \multirow{2}{*}{72.231} & FEM & 11.0842 & 0.6479 & 10.3362 & 0.6652 \\
& & & & EXP & 11.4332 & 3.6808 & 10.6627 & 3.7077 \\
\hline
\end{tabular}

\subsection{Optimization and results}

The problem defined by Eqs. (20)-(24) is an optimization problem with nonlinear constraints. In the traditional methodology, the objectives defined in Eq. (20) are generally converted into a single objective function by using different weighting factors. However, this methodology can only obtain a single optimal solution, and the determination of the weighting factors depends heavily on experiences. While the multi-objective optimization treats all the objectives separately and delivers a set of optimal solutions form the Pareto frontier. A Pareto optimal solution is one that any improvement in an objective will cause degradation in other objectives, and all Pareto optimal solutions are considered equally good.

In this paper, NSGA-II is adopted to find the Pareto frontier of the multi-objective optimization problem. NSGA-II is a multi-objective exploratory technique, which has been successfully used in many engineering optimization jobs (Nikkhah Kashani and Rafiei, 2014; Ma et al., 2013; Kelaiaia et al., 2012) and is becoming one of the most popular methods to multi-objective problems. For the current work, we use a population size of 100 , a number of generations of 100 , and a mutation probability of 0.3 . All possible and optimal solutions which considering the three objectives simultaneously are shown in Fig. 9. The results indicate that the algorithm was able to find the Pareto front with good distribution.

Table 2 lists 6 representative optimal solutions ranked by motion range $\theta_{\max }$, where the index $k$ is the rank of $\theta_{\max }$ in the Pareto solution set. At the extreme point $f_{1}$, the ellipse-parabola shaped flexure hinge has the minimum motion range but higher rotation precision and relative compliance. While at the other extreme point $f_{6}$, the flexure hinge has the maximum motion range but lower rotation precision and relative compliance. All the other points are intermediate optimal solutions. The designers can select the optimal geometric parameters from the obtained Pareto solution set based on practical requirements.

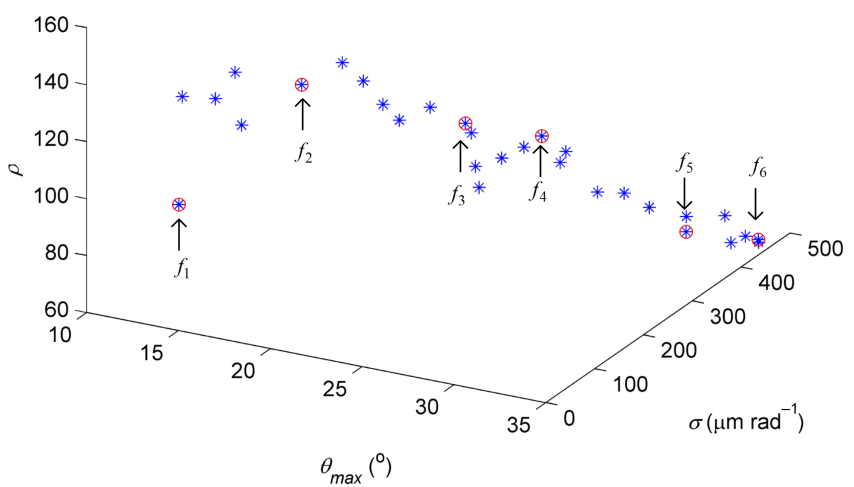

Figure 9. Pareto front obtained by NSGA-II.

\section{Conclusion}

A novel type of superelastic flexure hinge, called ellipseparabola shaped flexure hinge, is proposed in this paper to achieve optimal comprehensive performance. The ellipseparabola shaped flexure hinge is constructed by an ellipse arc and a parabola curve and smoothly connected at the intersection. The static response of the hinge is solved by non-prismatic beam elements which considered the geometric and material nonlinearities. Finite element analysis and experiment tests proved the accuracy and efficiency of the proposed method. The maximum relative error of CRM is within $1.5 \%$ and $7 \%$ compared to the AFE and the EXP respectively. A multi-objective optimization aims to maximize the motion range and the relative compliance of the ellipseparabola shaped flexure hinge as well as to minimize the relative rotation error during the deformation is conducted. And the Pareto frontier is found by using the NSGA-II algorithm. The optimization methodology presented in this article can be used and extended in the design process of other shaped superelastic flexure hinges. 
Table 2. The optimal solutions.

\begin{tabular}{ccccccccc}
\hline \multirow{2}{*}{ Point } & $k$ & \multicolumn{3}{c}{ Geometric parameter } & & \multicolumn{3}{c}{ Performance } \\
\cline { 3 - 4 } \cline { 7 - 8 } & & $l(\mathrm{~mm})$ & $e(\mathrm{~mm})$ & $\varphi_{\mathrm{t}}(\mathrm{rad})$ & & $\theta_{\max }\left({ }^{\circ}\right)$ & $\gamma\left(\mu \mathrm{m} \mathrm{rad}^{-1}\right)$ & $\rho$ \\
\hline$f_{1}$ & 1 & 10.179 & 2.673 & -1.498 & & 13.810 & 45.738 & 97.296 \\
$f_{2}$ & 6 & 14.899 & 4.912 & -1.393 & & 19.645 & 77.875 & 142.900 \\
$f_{3}$ & 12 & 32.484 & 4.981 & -1.146 & & 26.347 & 161.207 & 127.878 \\
$f_{4}$ & 18 & 11.845 & 4.969 & -1.456 & & 29.093 & 214.126 & 120.615 \\
$f_{5}$ & 24 & 8.673 & 4.950 & -1.401 & & 32.747 & 372.203 & 72.782 \\
$f_{6}$ & 30 & 20.893 & 4.981 & -1.236 & & 34.399 & 457.984 & 61.899 \\
\hline
\end{tabular}

Acknowledgements. This work was supported by National Natural Science Foundation of China under Grant No. 51475113, Natural Science Foundation of Heilongjiang Province under Grant No. E2015006, the State Key Lab of Self-planned Project under Grant No. SKLRS201501A03, and the Programme of Introducing Talents of Discipline to Universities under Grant No. B07018.

Edited by: G. Hao

Reviewed by: two anonymous referees

\section{References}

Chen, G., Shao, X., and Huang, X.: A new generalized model for elliptical arc flexure hinges, Rev. Sci. Instrum., 79, 095103, doi:10.1063/1.2976756, 2008.

Chen, G., Liu, X., Gao, H., and Jia, J.: A generalized model for conic flexure hinges, Rev. Sci. Instrum., 80, 055106, doi:10.1063/1.3137074, 2009.

Chen, G., Liu, X., and Du, Y.: Elliptical-Arc-Fillet Flexure Hinges: Toward a Generalized Model for Commonly Used Flexure Hinges, J. Mech. Design, 133, 81002, doi:10.1115/1.4004441, 2011

Criesfield, M. A.: Nonlinear finite element analysis of solids and structures, Volume 1: Essentials, Wiley, Location: New York, 1991.

De Bona, F. and Gh Munteanu, M.: Optimized Flexural Hinges for Compliant Micromechanisms, Analog Integr. Circ. S., 44, 163174, 2005.

Desroches, R. and Delemont, M.: Seismic retrofit of simply supported bridges using shape memory alloys, Eng. Struct., 24, 325332, 2002.

Du, Z., Shi, R., and Dong, W.: A Piezo-Actuated High-Precision Flexible Parallel Pointing Mechanism: Conceptual Design, Development, and Experiments, IEEE T. Robot., 30, 131-137, 2014.

Friedrich, R., Lammering, R., and Rösner, M.: On the modeling of flexure hinge mechanisms with finite beam elements of variable cross section, Precis. Eng., 38, 915-920, 2014.

Hesselbach, J. and Raatz, A.: Pseudo-elastic Flexure-Hinges in Robots for Micro Assembly, in: Proc. SPIE Microrobotics and Microassembly II, Boston, USA, 4194, 157-167, doi:10.1117/12.403696, 2000.

Kelaiaia, R., Company, O., and Zaatri, A.: Multiobjective optimization of parallel kinematic mechanisms by the genetic algorithms, Robotica, 30, 783-797, 2012.
Kim, H., Kim, J., Ahn, D., and Gweon, D.: Development of a Nanoprecision 3-DOF Vertical Positioning System With a Flexure Hinge, IEEE T. Nanotechnol., 12, 234-245, 2013.

Liew, K. M., Ren, J., and Kitipornchai, S.: Analysis of the pseudoelastic behavior of a SMA beam by the element-free Galerkin method, Eng. Anal. Bound. Elem., 28, 497-507, 2004.

Lobontiu, N., Paine, J. S. N., Malley, E. O., and Samuelson, M.: Parabolic and hyperbolic flexure hinges: flexibility, motion precision and stress characterization based on compliance closedform equations, Precis. Eng., 26, 183-192, 2002a.

Lobontiu, N., Paine, J. S. N., Garcia, E., and Goldfarb, M.: Design of symmetric conic-section flexure hinges based on closedform compliance equations, Mech. Mach. Theory, 37, 477-498, 2002b.

Ma, M., Sun, B., Wang, J., and Shi, J.: Multi-objective optimization design for leg mechanism of hydraulic-actuated quadruped robot, Journal of Beijing Institute of Technology (English Edition), 22, 12-19, 2013.

Nikkhah Kashani, H. and Rafiei, S. M. R.: Optimal Control of Active Power Filters using Fractional Order Controllers Based on NSGA-II Optimization Method, Int. J. Elec. Power, 63, 10081014, 2014.

Pacoste, C. and Eriksson, A.: Beam elements in instability problems, Comput. Method. Appl. M., 144, 163-197, 1997.

Paros, J. M. and Weisbord, L.: How to design flexure hinges, Mach. Des., 37, 151-156, 1965.

Pham, H. and Chen, I.: Stiffness modeling of flexure parallel mechanism, Precis. Eng., 29, 467-478, 2005.

Shi, R. C., Dong, W., and Du, Z. J.: Design methodology and performance analysis of application-oriented flexure hinges, Rev. Sci. Instrum., 84, 075005, doi:10.1063/1.4813252, 2013.

Tian, Y., Shirinzadeh, B., Zhang, D., and Zhong, Y.: Three flexure hinges for compliant mechanism designs based on dimensionless graph analysis, Precis. Eng., 34, 92-100, 2010.

Wang, R., Zhou, X., and Zhu, Z.: Development of a novel sort of exponent-sine-shaped flexure hinges, Rev. Sci. Instrum., 84, 095008, doi:10.1063/1.4821940, 2013.

Wu, Y. and Zhou, Z.: Design calculations for flexure hinges, Rev. Sci. Instrum., 73, 3101-3101, 2002.

Yong, Y. K., Lu, T., and Handley, D. C.: Review of circular flexure hinge design equations and derivation of empirical formulations, Precis. Eng., 32, 63-70, 2008. 